\title{
Development of superhydrophobic electrospun fibrous membrane of polymers of intrinsic microporosity (PIM-2)
}

\author{
Bekir Satilmis $^{\mathrm{a}, \mathrm{b}, *}$, Tamer Uyar ${ }^{\mathrm{a}, *}$ \\ ${ }^{a}$ Institute of Materials Science \& Nanotechnology, UNAM-National Nanotechnology Research Center, Bilkent University, Ankara 06800, Turkey \\ ${ }^{\mathrm{b}}$ Department of Chemistry, Faculty of Science and Arts, Ahi Evran University, Kirsehir 40100, Turkey
}

\section{A R T I C L E I N F O}

\section{Keywords:}

Polymers of Intrinsic Microporosity

PIM-2

Electrospinning

Membrane

Nanofibers

Adsorption

Superhydrophobic

Oil removal

\begin{abstract}
A B S T R A C T
Polymers of intrinsic microporosity (PIMs) are increasingly recognized as a potential membrane material for adsorption and separation applications due to their permanent porosity and solution processability. PIM-2 can be produced using commercially available 5,5',6,6'-Tetrahydroxy-3,3,3',3'-tetramethyl-1,1'-spirobisindane and decafluorobiphenyl monomers in the powder form. It possesses considerable amount of fluorine in the polymer backbone and this feature could provide significant hydrophobicity to polymer. This research aims to investigate the fabrication of self-standing PIM-2 fibrous membranes by electrospinning method to introduce a useful material for adsorption applications. Electrospinning was performed using tetrachloroethane as a solvent and beadfree, uniform fibers were produced as confirmed by SEM imaging. Average fiber diameter was calculated as $5.5 \pm 1.5 \mu \mathrm{m}$ for a self-standing fibrous membrane of PIM-2. Structural characterization was conducted using FT-IR, NMR and XPS spectroscopies showing the purity of pristine powder and fibrous membrane of PIM-2. Thermal stability of PIM-2 fibrous membrane was investigated using TGA and it shows no discernible weight loss below $450{ }^{\circ} \mathrm{C}$. The porosity of fibrous membrane was investigated by $\mathrm{N}_{2}$ adsorption/desorption measurements that indicates significant microporosity with $\sim 600 \mathrm{~m}^{2} \mathrm{~g}^{-1}$ BET surface area. In addition, the hydrophobicity of PIM-2 was tested by water contact angle measurements, showing $155 \pm 6^{\circ}$ WCA, indicating superhydrophobicity owing to rough surface and high fluorine content. Consequently, the combination of straightforward synthesis, solution processability, high thermal stability, high surface area, and superhydrophobicity makes PIM-2 a promising candidate for adsorption applications. Therefore, it was successfully employed in organic and oil adsorption. Fibrous membranes of PIM-2 has shown up to $2200 \pm 100 \%$ and $1900 \pm 100 \%$ weight gain after in contact with silicon oil and DMSO respectively. In addition, dense membrane of PIM-2 was prepared by solvent casting method and the uptake ability was compared with fibrous membrane showing that fibrous form is more convenient for liquid adsorption applications.
\end{abstract}

\section{Introduction}

Recently, Polymers of Intrinsic Microporosity (PIMs) have been introduced as a potential membrane material for adsorption and separation applications $[1,2]$. This novel class of polymers exhibit high free volume as a result of their rigid and contorted structures which limit the rotational freedom and provide packing inefficiency [3]. When PIMs were first introduced, six different PIMs were reported (PIM-1 thru PIM6) using spiro monomers and fluoroarenes [1]. PIM-1, which is produced from 5,5',6,6'-tetrahydroxy-3,3,3', $3^{\prime}$-tetramethyl-1, $1^{\prime}$-spirobisindane (TTSBI) and tetrafluorophthalonitrile (TFTN) monomers, is the first introduced member of this class of polymers. It has gained most of the attention due to its high surface area, high molecular weight, solubility in common organic solvents (tetrahydrofuran, chloroform and etc.) and the simplicity of the synthesis as it was directly produced from commercially available monomers [1,4-6]. PIM-1 has been used in the form of powder [7], film [8-10] and fiber [11,12] due to the solution processability. Particularly, it has shown outstanding gas separation performance [13]. While other five PIMs also show similar structural properties like PIM-1, they have not been successfully produced in the membrane forms which limit their effective use and their possible applications. Although several other PIMs have also been introduced lately $[14,15]$ and have been employed in a number of applications such as gas separation, adsorption, and filtration, they usually require more complicated synthesis paths, efforts, time and cost in the production compared to first generation of PIMs (PIM-1 thru PIM-6) [16-18]. Despite the significant properties of PIM-2 which is produced directly from commercially available TTSBI and

\footnotetext{
* Corresponding authors at: Department of Chemistry, Faculty of Science and Arts, Ahi Evran University, Kirsehir 40100, Turkey (B. Satilmis).

E-mail addresses: bekir.satilmis@ahievran.edu.tr (B. Satilmis), uyar@unam.bilkent.edu.tr (T. Uyar).
} 
(a)<smiles>COc1cc2c(cc1O)C1(CC(C)(C)c3cc(O)c(O)cc31)CC2(C)C</smiles>

TTSBI<smiles>Fc1c(F)c(F)c(-c2c(F)c(F)c(F)c(F)c2F)c(F)c1F</smiles>

DFBP

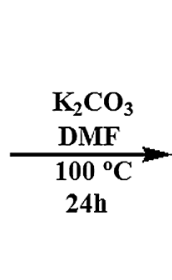

(O)<smiles>CCCc1cc2c(cc1CC)Oc1c(F)c(F)c(-c3c(F)c(F)c(OC)c(F)c3F)c(F)c1O2</smiles>

PIM-2

(b)

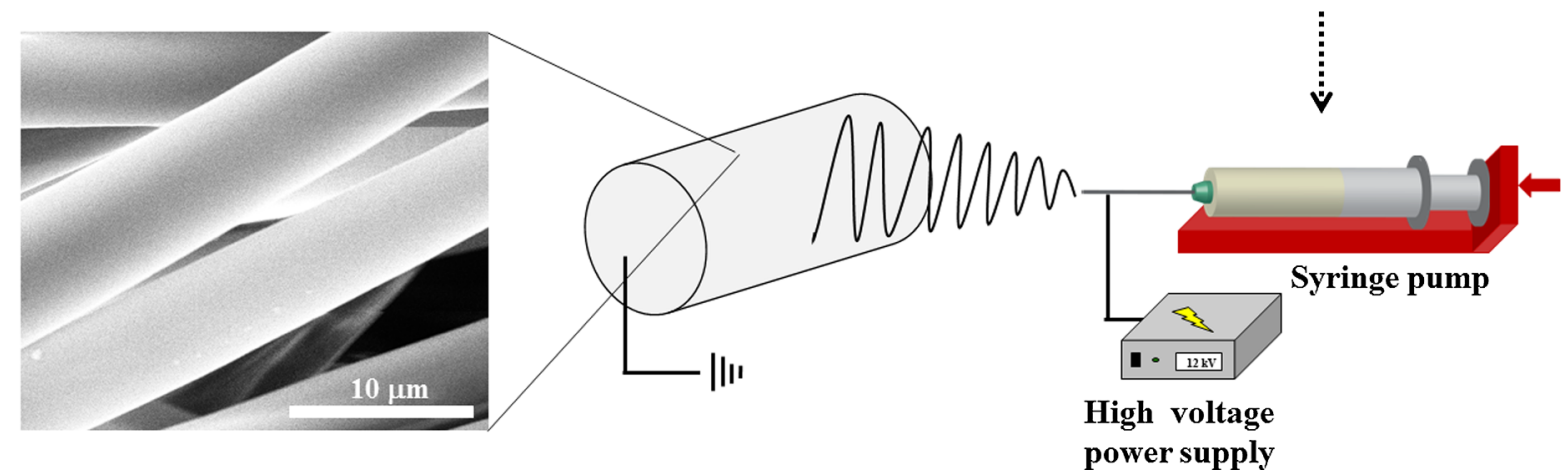

Fig. 1. (a) Chemical structures of monomers and synthesis path of PIM-2 (b) Schematic representation of electrospinning of PIM-2 fibers.

decafluorobiphenyl monomers; the importance of PIM-2 is still somehow overlooked in the literature where only very limited research has been performed to investigate the PIM-2. Budd et al. [1] showed that the BET surface area of PIM-2 is around $600 \mathrm{~m}^{2} \mathrm{~g}^{-1}$ but they did not manage to obtain high enough molecular weight to produce membrane (film) form. Recently, a systematic study has been reported by Sato et al.[19] and they have provided an important data for the synthesis of PIM-2. Even though they have performed a detailed study in synthesis and characterization by aiming to reach high molecular weight polymer, they were not able to produce a membrane form of PIM-2. Hence, previous studies have only provided some insights into synthesis of PIM-2 powder that limit the practical use of this highperformance polymer. Regarding the high BET surface area and high fluorine content of PIM-2, successful production of PIM-2 membrane may trigger a growing body of research in adsorption and separation applications.

In this study, we have produced self-standing fibrous membrane of PIM-2 by electrospinning technique. Electrospinning is a simple and cost-effective method to produce high surface area fibrous membranes from polymeric materials [20-22] which are quite suitable for filtration and adsorption applications due to their high surface area and their hydrophobicity [23-27]. While polymeric fibrous membranes could be used as a self-standing membrane they can also be used as a polymeric support for various applications including photocatalysis and sensor applications [28-30]. Indeed, such fibrous membranes could also be employed for water treatment in the membrane form. Some successful examples of electrospun fibrous membranes were reported for this purpose such as polyvinylidenefluoride-co-hexafluoropropylene [31], poly(tetrafluoroethylene-co-hexafluoropropylene-co-vinylidene

fluoride) [32] and polysulfone/polydimethylsiloxane membranes [33]. On the other hand, PIMs were usually employed in the dense membrane form due to their high gas and vapor sorption inside the polymer matrix [34-37]. Recently, a few studies have also been reported showing the outstanding performance of electrospun fibrous membrane of PIM-1 and modified PIM-1s in adsorption and separation applications $[11,27,30,38-43]$. Yet, to the best of our knowledge, the present study is the first demonstration of successful production of electrospinning of self-standing PIM-2 fibrous membrane, and also comparative studies on the structural properties of electrospun PIM-2 fibrous membrane with powder form of PIM-2 were performed. Afterwards, the adsorptive properties of PIM-2 fibrous membrane were evaluated for an organic and oil adsorption. We have also managed to produce stable film form of PIM-2 and compared the adsorption ability with fibrous form.

\section{Experimental}

\subsection{Materials}

5,5',6,6'-Tetrahydroxy-3,3,3',3'-tetramethyl-1,1'-spirobisindane (TTSBI, 98\%, Alfa Aeser) were purified according to previous method [44]. Decafluorobiphenyl (DFBP, 99\%, Sigma Aldrich) was used as received. Anhydrous potassium carbonate $\left(\mathrm{K}_{2} \mathrm{CO}_{3}, 99.0 \%\right.$, Alfa Aeser) was grinded before use. 1,1,2,2, tetrachloroethane (TCE, 98\%), methanol $(\mathrm{MeOH}, \geq 99.9 \%)$ and anhydrous dimethylformamide (DMF, 99.8\%) were obtained from Sigma Aldrich. Sunflower oil (Yudum, obtained from a local shop), pump oil (Edwards 19, ultragrade vacuum oil), and silicone oil were used as received. DMF ( $\geq 99 \%)$, dimethylsulfoxide (DMSO, $\geq 99 \%$ ), hexane ( $\geq 95 \%$ ), 1,4 dioxane ( $\geq 99 \%$ ), chloroform $\left(\mathrm{CHCl}_{3}\right.$, $\geq 99 \%$ contains ethanol as stabilizer), toluene ( $\geq 99.5 \%$ ) were obtained from Sigma Aldrich.

\subsection{Synthesis of PIM-2}

Synthesis of PIM-2 was performed similar to method described by Sato et al. [19] TTSBI $(2.0427 \mathrm{~g}, 6 \mathrm{mmol})$ and $\mathrm{K}_{2} \mathrm{CO}_{3}(0.833 \mathrm{~g}, 6 \mathrm{mmol})$ were dissolved in $12 \mathrm{~mL}$ anhydrous DMF under argon atmosphere in a 250-mL three-necked round bottom flask which was equipped with reflux condenser, and magnetic stirring-bar. The mixture was heated $100{ }^{\circ} \mathrm{C}$ for half an hour and then $2.0 \mathrm{~g}$ DFBP which was dissolved in $58 \mathrm{~mL}$ anhydrous DMF was added drop-wisely with the help of syringe pump ( $12 \mathrm{~mL}$ plastic syringe and $20 \mathrm{~mL} \mathrm{~h}^{-1}$ flow rate). The reaction was kept at $100^{\circ} \mathrm{C}$ for $24 \mathrm{~h}$ which gives off-white suspension. The suspension was poured into $150 \mathrm{~mL}$ aqueous $\mathrm{HCl}(5 \% v / v)$. It was filtered under vacuum by sinter funnel and dried at $150{ }^{\circ} \mathrm{C}$ for overnight. Crude product was stirred in methanol for $2 \mathrm{~h}$ then filtered again. The solid dried at $110{ }^{\circ} \mathrm{C}$ overnight. Off-white fine powder was obtained with $85 \%$ yield $(3.02 \mathrm{~g})$. Fig. 1 a shows the chemical structures of monomers and synthesis path of PIM-2. Also, visual representation of PIM-2 synthesis is provided in Fig. S1 in supporting information. 


\subsection{Electrospinning of PIM-2 fibers}

PIM-2 powder was dissolved in tetrachloroethane (TCE) at a concentration of $43 \%$ and it was stirred at room temperature for $5 \mathrm{~h}$ to ensure complete dissolution. The solution was pumped out of a $1 \mathrm{~mL}$ syringe through $18 \mathrm{G}$ blunt needle with the help of a syringe pump (KD Scientific, KDS 101). Metal plate collector was covered by an aluminum foil then placed across the syringe to collect microfibers. The electrospinning process is presented in Fig. 1b. The applied electrospinning parameters were: flow rate of the polymer solution; $0.6 \mathrm{~mL} \mathrm{~h}^{-1}$, applied voltage; $12 \mathrm{kV}$ and tip to collector distance; $18 \mathrm{~cm}$, rotation speed; $1000 \mathrm{rpm}$. Then, the collected fibers were detached from the aluminum surface and dried in an oven at $110{ }^{\circ} \mathrm{C}$ for overnight.

\subsection{Preparation of PIM-2 dense membrane (film)}

PIM-2 (0.2 g) powder was dissolved in chloroform $(5 \mathrm{~mL})$ and it was stirred overnight. The solution was poured into petri dish and the solvent was evaporated slowly at room temperature.

\subsection{Methods}

Gel permeation chromatography (GPC) analysis was performed with an Agilent GPC instrument. ZORBAX PSM 300-S column was employed using pure THF as the eluent at a flow rate of $1 \mathrm{mg} \mathrm{mL}^{-1}$. Bruker Vertex 70 FT-IR Spectrometer was utilized for investigating the chemical structure and functional groups of PIM-2 in transmittance mode. ${ }^{1} \mathrm{H}$ NMR and ${ }^{19} \mathrm{~F}$ NMR measurements were conducted using Bruker DPX$400 \mathrm{MHz}$ NMR spectrometer using $\mathrm{CDCl}_{3}$ as a solvent. Electrospun fibers were characterized for their morphology via scanning electron microscopy (SEM) using FEI Quanta 200 FEG instrument. The samples were sputtered with gold prior to imaging. The micrographs were analyzed using ImageJ software to measure the diameters of the fibers. The XPS spectra of the samples were recorded with a Thermo Fisher Scientific K-alpha XPS Spectrometer which is equipped with monochromatic Al K-alpha source. Thermogravimetric analysis was performed using TA Q500 (TA instruments). The samples were heated from 30 to $600{ }^{\circ} \mathrm{C}$ at a heating rate of $20^{\circ} \mathrm{C} \mathrm{min}^{-1}$ with nitrogen as purge and balance gas. Porosity of the samples were determined using $\mathrm{N}_{2}$ sorption isotherms at $77 \mathrm{~K}$ obtained by Quantachrome Autosorb iQ gas sorption analyser. Samples were first degassed under under high vacuum for $16 \mathrm{~h}$ at $120^{\circ} \mathrm{C}$ prior to analysis. Water contact angles $(\theta)$ were measured on an OCA 20 goniometer (Dataphysics, Germany). $4 \mu \mathrm{L}$ water droplets were formed in open air at room temperature. Then Laplace-Young fitting was used to obtain WCA values. The mean values were obtained from eight different spots in the same sample. Adsorption of organic compounds and oils were performed using fibrous membranes $(1 \times 2 \mathrm{~cm}, \sim 10 \mathrm{mg})$ which were immersed in organic liquids for an hour and then taken out for weight measurements which were measured immediately to avoid evaporation. The excess liquid on the membrane surface was wiped-off by touching the vial surface without squeezing or using tissue. Also, dense membranes $(\sim 10 \mathrm{mg})$ were subjected to same conditions for chosen solvents and oils. The percent weight gain was calculated using Eq. (1).

$\%$ weight gain $=\frac{m_{1}-m_{0}}{m_{0}} \times 100$

where $\mathrm{m}_{0}$ and $\mathrm{m}_{1}$ are the weights of the membranes before and after adsorption, respectively. All measurements were performed as duplicates to increase the reliability of the measurement.

The density ( $\rho$ ) of PIM-2 samples were measured by gravimetric method according to Eq. (2) using dry samples. Void volumes of samples were calculated using Eq. (3) where $V_{\text {void, }} V_{\text {total }}$, and $V_{\text {polymer }}$ are void volume of the membrane, total volume of the membrane after sorption and dry polymer volume of the membrane, respectively. Also, adsorb liquid volume ( $\left.V_{\text {liquid }}\right)$ was calculated based on the Eq. (4) to compare the results. $m_{\mathrm{dry}}$ and $m_{\mathrm{wet}}$ are the mass of the membrane before and after sorption and $\rho_{\text {liquid }}$ is the density of the sorbed liquid. Note that here, membranes were pressed with tissue to remove the excess liquid from the surface.

$\rho_{\text {polymer }}\left(\mathrm{g} \mathrm{cm}^{-3}\right)=\frac{m_{\text {polymer }}}{(\text { Area } \times \text { Thickness })}$

$V_{\text {void }}=V_{\text {total }}-V_{\text {polymer }}=($ Area $\times$ Thickness $)-\left(\frac{m_{\text {polymer }}}{\rho_{\text {polymer }}}\right)$

$V_{\text {liquid }}=\frac{\left(m_{\text {wet }}-m_{\text {dry }}\right)}{\rho_{\text {liquid }}}$

\section{Results and discussion}

Synthesis and characterization of linear PIM-1 is now well-documented $[1,6]$. PIM-1 is a solution processable material and it was used in a number of papers in a wide range of applications such as adsorption, gas separation, pervaporation, nanofiltration and catalysis. [34,45-48]. However, the synthesis and characterization of other PIM polymers which were reported in the original publication [1] are not well studied either due to difficult reaction conditions or the low performance of resulting polymers compared to PIM-1. Budd et al. [1] first reported 6 type of PIM polymers but except PIM-1, the first polymer, others produced in a low molecular weight. Recently, Sato et al. [19] have reported a detailed study for the synthesis of PIM-2 which is produced from TTSBI and DFBP monomers in dimethylformamide at $100{ }^{\circ} \mathrm{C}$ for $24 \mathrm{~h}$. The obtained PIM-2 has decent molecular weight $\left(M_{\mathrm{n}}: 13500\right)$ and relatively low PDI (4.4). The PIM-2 was obtained in the form of yellow powder and it was soluble in THF and chloroform which makes it a solution processable. However, they did not manage to obtain a useful membrane/film form of PIM-2 due to its low molecular weight which limited their study only the synthesis and characterization of PIM-2 as powder form. Therefore, we have aimed to synthesize PIM-2 by the same method with a slight modification. After the given condition s applied, we have obtained off-white powder which was soluble in THF, chloroform and toluene. The polymer exhibits bimodal GPC curve as shown in Fig. S2 in supporting information which displays oligomeric structures and low molar mass polymeric structures having $M_{\mathrm{n}}$ : 5800 and 4.7 PDI values. Obtaining higher molecular weight of PIM-2 seems difficult since cyclic structures may inhibit linear chain growing of this kind of polymer [19]. After measuring GPC, we have characterized the polymer with FT-IR and NMR spectroscopies to ensure the purity of PIM-2. Fig. 2 shows the FT-IR spectrum of PIM-2 powder. Aliphatic stretches of C-H peaks can be seen below $3000 \mathrm{~cm}^{-1}$.

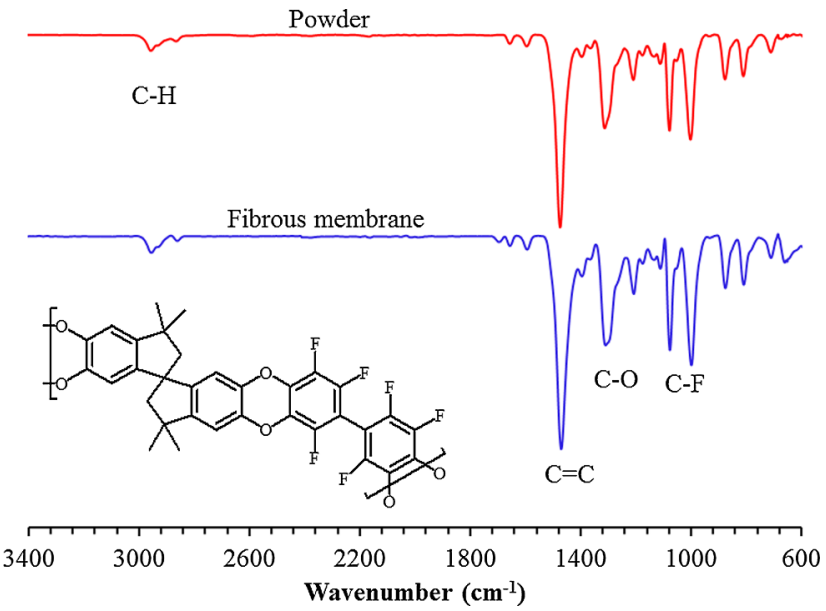

Fig. 2. FT-IR spectra of PIM-2; powder form and electrospun fibrous membrane. 
(a)
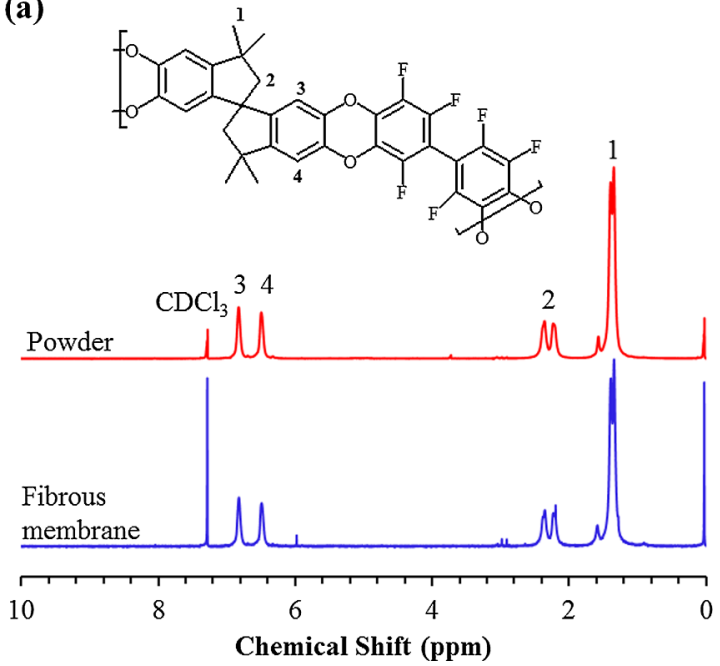

(b)

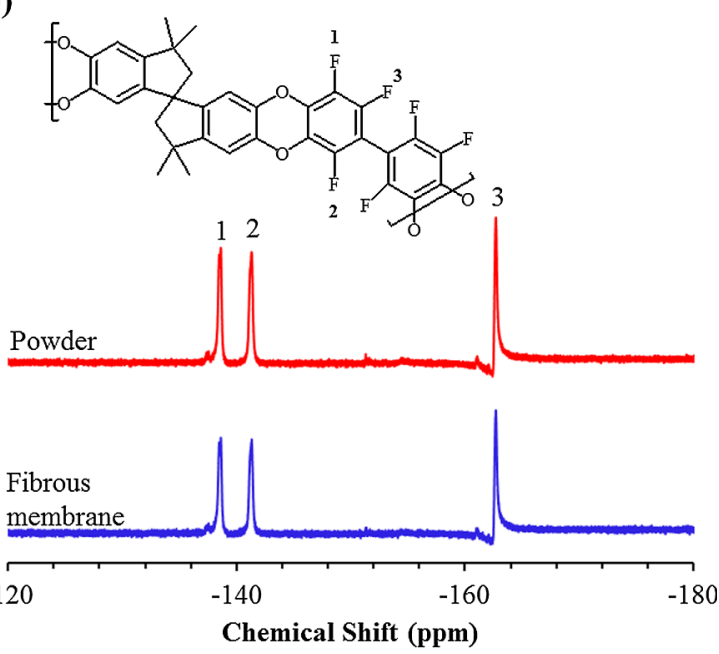

Fig. 3. (a) ${ }^{1} \mathrm{H}$ NMR and (b) ${ }^{19} \mathrm{~F}$ NMR spectra of PIM-2 powder and fibrous membrane (the samples were dissolved in $\mathrm{CDCl}_{3}$ ).

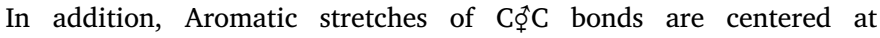
$1469 \mathrm{~cm}^{-1}$ along with $\mathrm{C}-\mathrm{O}\left(1299 \mathrm{~cm}^{-1}\right)$ and $\mathrm{C}-\mathrm{F}$ (995 and $1076 \mathrm{~cm}^{-1}$ ) stretches. Moreover, the absence of some peaks also indicates the purity and success of polymerization as reported by Sato et al. [19]. The absence of terminal TTSBI and DFBP units in the polymer structure is confirmed by the absence of phenolic peaks $\left(3400 \mathrm{~cm}^{-1}\right)$ and the peaks at $988(\mathrm{C}-\mathrm{F}), 797(\mathrm{C}-\mathrm{H})$, and $719(\mathrm{C}-\mathrm{F})$ $\mathrm{cm}^{-1}$. Furthermore, the absence of peaks at $1149 \mathrm{~cm}^{-1}$, shows that no open ether linkages present in our synthesized PIM-2.

${ }^{1} \mathrm{H}$ NMR spectrum of PIM-2 powder is depicted in Fig. 3a which shows characteristic PIM peaks originate from spiro-monomer that reveals 4 peaks; 2 aromatics and 2 aliphatic peaks and the ratio of them is 1 to 4 . In addition, due to the abundance of fluorine groups in PIM-2, we have performed ${ }^{19} \mathrm{~F}$ NMR which shows three district fluorine environments regardless of its form since PIM-2 may polymerize in four different configurations [19]. These aromatic peaks are located at $-162.7,-141.4$ and $-138.7 \mathrm{ppm}$. The first one is considered as the para positioned $\mathrm{F}$ atoms with respect to the oxygen atom.

Investigating different forms of PIMs is a growing interest since they can be employed in a number of applications. Therefore, after confirming the purity of PIM-2 powder, electrospinning was conducted. As the solubility of PIM-2 is similar to PIM-1, the electrospinning of PIM-2 was achieved using the same spinning solvent; tetrachloroethane $[11,30]$. Recently, we have reported the electrospinning of PIM-1, which has $M_{\mathrm{w}} \sim 190,000$ and narrow PDI 1.78, was electrospun using $23 \%(w / v)$ polymer concentration in tetrachloroethane [11]. Therefore, first by considering the low molecular weight of PIM-2, 38\% $(w / v)$ PIM2 concentration was prepared to obtain a continues jet, and a stationary collector was used for collecting random fibers but wetting and film forming was observed due to the high boiling point of electrospinning solvent (TCE bp: $147^{\circ} \mathrm{C}$ ). Afterwards, a rotating collector was used to accelerate the evaporation of the solvent during electrospinning process. At low rotation speed $(100 \mathrm{rpm})$, fibers started to form but the obtained fibers were highly interlaced and fused each other as shown in Fig. 4a. When the rotation speed increased to $1000 \mathrm{rpm}$, highly aligned fibers were obtained and the observed morphology was beads-on-string (Fig. 4b), indicating that higher concentration is required for bead-free fibers. Therefore, the concentration was increased to $43 \%(w / v)$ which resulted in bead-free and aligned fibers as displayed in Fig. 4c. The averaged fiber diameter was calculated as $5.5 \pm 1.5 \mu \mathrm{m}$ and the fiber diameter distribution of the PIM-2 fibrous membrane is presented in Fig. 4d. The off-white PIM-2 fibers were obtained as self-standing fibrous membranes as shown in Fig. 4e. It also shows significant bending ability which depicts the structural stability of the membrane.
After producing self-standing fibrous membrane form of PIM-2, the purity of the sample was compared with powder form by FTIR and NMR as shown in Fig. 2 and Fig. 3. Fibrous membrane shows identical peaks with powder form in FT-IR and NMR spectra. In addition, no obvious signal was observed for the electrospinning solvent (TCE) in ${ }^{1} \mathrm{H}$ NMR spectrum confirming that the solvent was completely removed from the PIM-2 fibers. Following that, the structural characterization was further performed with XPS analysis. Due to the high fluorine content, elemental analysis is not completely reliable as it is difficult to observe oxygen and fluorine contents. On the other hand, XPS spectroscopy is a useful technique to characterize the polymers. XPS spectra of powder and fibrous membrane of PIM-2 are displayed in Fig. S3 in supporting information. Three distinct peaks can be seen for both powder and fibrous membrane which are $\mathrm{C}(285 \mathrm{eV}), \mathrm{O}(534 \mathrm{eV})$ and $\mathrm{F}(688 \mathrm{eV})$. Also, the fibrous membrane does not show any apparent chlorine peak from the spinning solvent $(199 \mathrm{eV})$. Moreover, the compositions of powder and the fibrous membrane can be seen in Table 1 that shows similar content for all atoms. However, considering the chemical formula of the polymer which is $\mathrm{C}_{33} \mathrm{H}_{20} \mathrm{~F}_{6} \mathrm{O}_{4}$, the obtained carbon content is slightly higher than theoretical values for both samples since the carbon contamination is a problem with PIM samples in XPS measurements $[43,49]$. Thus, we have correlated the data with theoretical values and it shows a good correlation as shown in Fig. S4 in supporting information.

Thermal properties of the powder and fibrous membrane were examined by TGA and they show almost identical degradation as depicted in Fig. 5. While they were stable up to $450^{\circ} \mathrm{C}$, backbone degradation occurs after this temperature similar to PIM-1 [11]. At $600^{\circ} \mathrm{C}$ still, over $65 \%$ of the polymer remained which exhibits the high thermal stability.

PIMs usually show a high surface area due to their rigid and contorted structure $[1,3]$. When PIM-2 was first reported, it showed $600 \mathrm{~m}^{2} \mathrm{~g}^{-1}$ BET surface area [1]. Therefore, we have tested $\mathrm{N}_{2}$ adsorption/desorption experiments to investigate the reproducibility of this result. Fig. 6 displays the $\mathrm{N}_{2}$ adsorption/desorption isotherms of PIM-2 powder and fibrous membrane. PIM-2 powder shows $615 \mathrm{~m}^{2} \mathrm{~g}^{-1}$ BET surface area (Table 1) which is almost the same as the reported data [1]. It shows type-I isotherm which denotes significant microporosity as it shows high uptake at low-pressure range. In addition, a hysteresis can be seen which suggests material has also mesoporous. Moreover, the PIM-2 fibrous membrane also maintains the surface area and microporosity showing $580 \mathrm{~m}^{2} \mathrm{~g}^{-1}$ BET surface area (Table 1) which is quite close to the data obtained for powder. However, similar to PIM-1 and hydrolyzed PIM-1 fibrous membranes [11,38], a slight reduction in BET surface area is observed for PIM-2 fibrous membrane 



Fig. 4. SEM images of electrospun PIM-2 fibers at (a) $38 \%(w / v)$ and $100 \mathrm{rpm}$ rotation speed, (b) $38 \%(w / v)$ and $1000 \mathrm{rpm}$ rotation speed (c) $43 \%$ ( $w / v)$ and 1000 rpm rotation speed. (d) Average fiber diameter distribution of PIM-2, and (e) Digital images of self-standing PIM-2 fibrous membrane.

compared to powder form. This slight reduction mostly occurs due to the methanol treatment strategy. While powder form was kept in methanol for $2 \mathrm{~h}$ to avoid the effect of synthesis history, fiber form was only treated with methanol for a short time to detach the fibers from aluminum surface. We have recently discussed this phenomena in detail in our previous report [43], revealing methanol treated PIM powders usually show higher surface area than that of the fibrous membranes since high boiling point spinning solvents could fill some of the pores in polymer matrix [43].

Compared to PIM-1[11] ( $\sim 760 \mathrm{~m}^{2} \mathrm{~g}^{-1}$ BET surface area), PIM-2 shows slightly lower surface area but the hydrophobicity of PIM-2 fibrous membrane could be higher than that of PIM-1 fibrous membrane

Table 1

Percent of atoms by XPS, BET surface area, micropore volume, micropore area, total pore volume and average pore diameter of PIM-2 powder and fibrous membrane.

\begin{tabular}{|c|c|c|c|c|c|c|c|c|}
\hline Sample & \multicolumn{3}{|c|}{ XPS (\% Atoms) } & $\begin{array}{l}\text { BET surface area } \\
-\left(\mathrm{m}^{2} \mathrm{~g}^{-1}\right)\end{array}$ & $\begin{array}{l}\text { Micropore volume } \\
\left(\mathrm{cm}^{3} \mathrm{~g}^{-1}\right)\end{array}$ & $\begin{array}{l}\text { Micropore area } \\
\left(\mathrm{m}^{2} \mathrm{~g}^{-1}\right)\end{array}$ & $\begin{array}{l}\text { Total pore volume } \\
\left(\mathrm{cm}^{3} \mathrm{~g}^{-1}\right)\end{array}$ & $\begin{array}{l}\text { Average pore diameter } \\
(\mathrm{nm})\end{array}$ \\
\hline Powder & 75.1 & 9.6 & 15.2 & 615 & 0.15 & 332 & 0.58 & 3.8 \\
\hline
\end{tabular}






Fig. 5. TGA curves of PIM-2 powder and fibrous membrane.

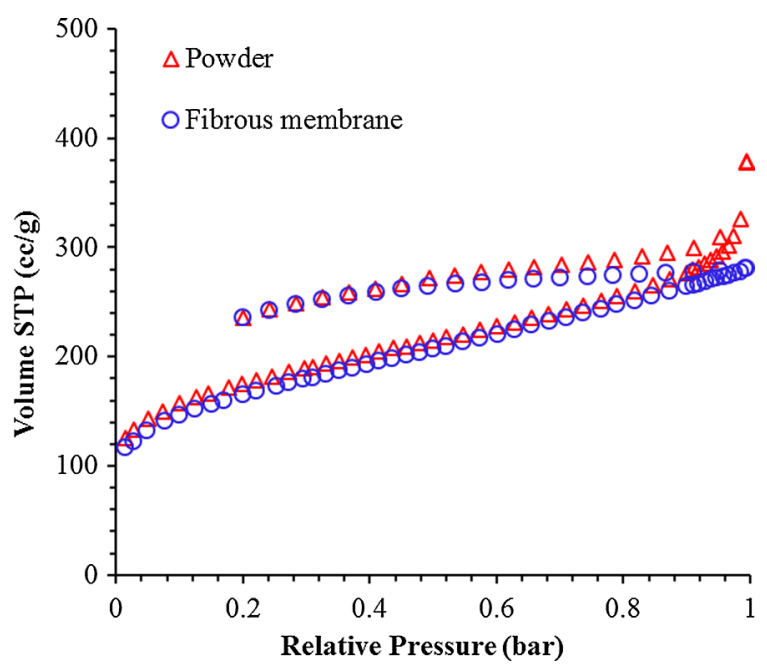

Fig. 6. $\mathrm{N}_{2}$ adsorption-desorption isotherms of PIM-2 powder and fibrous membrane.

(water contact angle; $\sim 130^{\circ}[11,39]$ ) since PIM-2 contains several fluorine groups in the backbone (Fig. 1a). Therefore, the water contact angle of PIM-2 was measured and it was found that PIM-2 fibrous membrane shows $155 \pm 6^{\circ}$ WCA which suggests that the electrospun PIM-2 fibrous membrane has a superhydrophobic characteristic. Fig. 7a exhibits the digital images of water droplets on PIM-2 fibrous membrane. Thus, as anticipated, high fluorine content along with a rough surface provides a greater water contact angle for PIM-2 fibrous membrane. In addition, although the molecular weight of our PIM-2

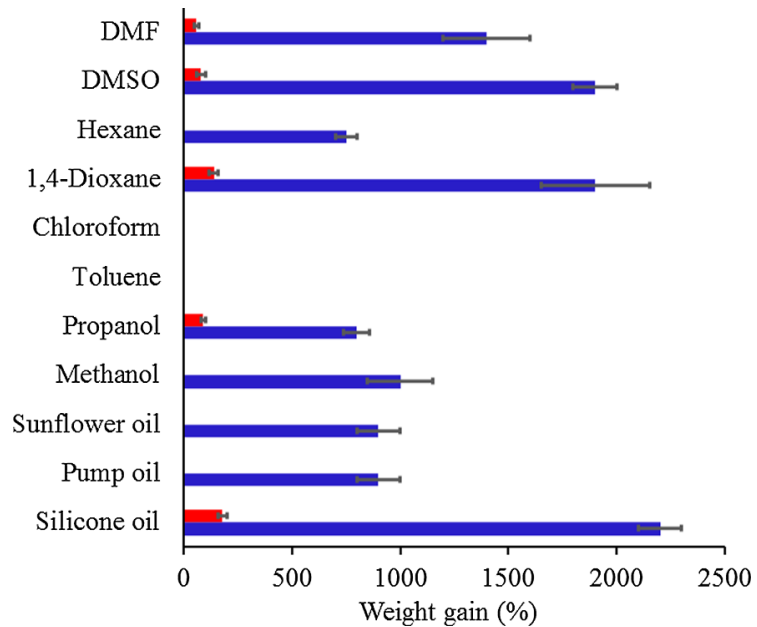

Fig. 8. The \% weight gain of PIM-2 fibrous membranes (blue) and dense membranes (red) after organic and oil adsorption. (For interpretation of the references to colour in this figure legend, the reader is referred to the web version of this article.)

polymer is lower than the reported PIM-2 by Sato et al. [19], we have managed to produce PIM-2 dense membrane (film) by solvent casting method in chloroform (Fig. S5 in supporting information). The success may only be related to the speed of evaporation or the personal experience for membrane preparation. Nevertheless, water contact angle of dense membrane was found as $93 \pm 3^{\circ}$, as displayed in Fig. $7 \mathrm{~b}$, revealing hydrophobicity is also presented in dense form of PIM-2.

High surface area and high hydrophobicity are prerequisite properties for an ideal adsorbent [50,51]. PIM-2 fibrous membrane shows both of these properties along with high structural stability in several organic solvents. Therefore, the adsorption ability of PIM-2 fibrous membrane was investigated using common organic solvents and oils. Fig. 8 displays the \% weight gain of PIM-2 fibrous membranes after in contact with organic solvents and oils. As stated previously, chloroform and toluene are good solvents for PIM-2, thus no data can be obtained for these solvents. However, for other common organic solvents, PIM-2 fibrous membrane showed considerable weight gain up to $\sim 2000 \%$ weight gain after in contact with solvents (DMSO and 1,4 dioxane). In a similar way, it has also showed high oil uptake $(2200 \pm 100 \%$ weight gain in silicon oil, Fig. 8). One surprising fact is, PIM polymers swell in alcohol and it supposed to adsorb more but the obtained values are lower than oil adsorption values. This is possibly because of the rapid evaporation of alcohols. In our previous report [27], hexamethylene diisocyanate (HMDI) crosslinked hydrolyzed PIM-1 fibrous membranes were also employed in a similar application and in this study PIM-2 fibrous membranes showed similar oil uptakes and hydrophobicity with those HDMI crosslinked hydrolyzed PIM-1 fibrous membranes. Yet,
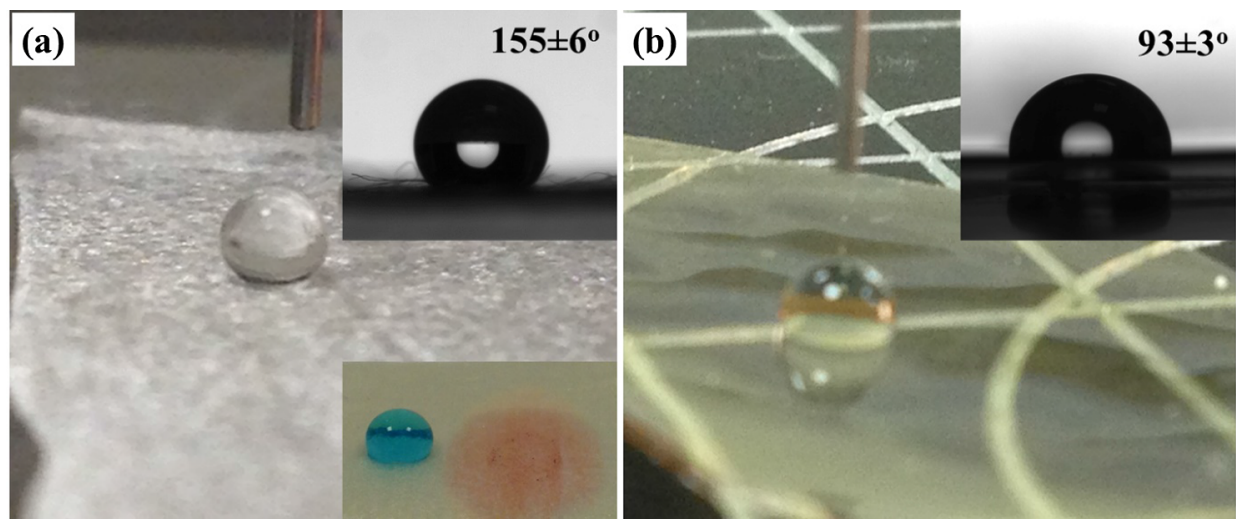

Fig. 7. Digital images of actual water droplets on (a) PIM-2 fibrous membrane, inset pictures are water contact angle images and oil and water droplets on PIM-2 fibrous membrane (water was colored by methylene blue and oil was colored by sudan-III), (b) PIM-2 dense membrane, inset picture is water contact angle image. (For interpretation of the references to colour in this figure legend, the reader is referred to the web version of this article.) 
hydrolyzed PIM-1 needed thermal treatment for crosslinking with HDMI whereas PIM-2 fibrous membrane itself shows considerable oil and organic uptake thanks to its high surface area and hydrophobicity.

In addition, uptake ability of PIM-2 dense membrane was evaluated under the identical conditions using some of the selected oils and organic solvents to compare with fibrous membrane, as displayed in Fig. 8. It can be seen that the adsorption by fibrous membrane is much higher than that of dense membrane due to the empty spaces (voids) in fibrous membrane. This result indicates that the adsorption mostly occurs in between the fibers but that is not the only place where it happens since dense membrane can also adsorb some liquid. To further investigate that first densities of the membranes were measured by using their dimensions and dry weights which gives $0.68 \pm 0.02$ and $1.24 \pm 0.02 \mathrm{~g} \mathrm{~cm}^{-3}$ for fibrous and dense membranes, respectively. Then membranes were soaked in methanol and void volumes were calculated using Eq. (3). The void volumes were found $22 \pm 3 \%$ and over $\sim 100 \%$ for dense and fibrous membranes. Additionally, the volume of the liquid adsorbed by a membrane (Eq. (4)) was calculated by using the mass difference and it gives $55 \pm 3 \%$ and $300 \%$ based on the dry volume of dense and fibrous membranes. These values were obtained after squeezing the membranes with tissue which is necessary to obtain some logic values otherwise the liquid volume is found much higher. Ideally, the data obtained from Eq. (3) (void volume) and Eq. (4) (liquid volume) should be identical but the data obtained from Eq. (4) are much higher than that of Eq. (3). The reason for this discrepancy is due to the swelling behavior of polymer in methanol. This behavior was also attempted to display visually in Fig. S6 in supporting information. Especially, fibrous membrane shows substantial increase in volume and methanol opens up the space in fibrous membrane. Nevertheless, one thing is certain that fibrous membrane has much greater porosity than that of dense membrane. The experiment was conducted using another solvent, hexane, to see whether we will obtain parallel results or not. For a dense membrane, we have found 25 (void volume) and $30 \%$ (liquid volume) which are similar to methanol data. However, for fibrous membrane this data was found as 20 (void volume) and $135 \%$ (liquid volume) based on the Eqs. (3) and (4), respectively. Considering all together, for all the measurements, membrane surfaces were cleared by tissue to remove the excess liquid but the real amount maintaining on the membranes are much greater than the void calculations. Aforementioned calculations only show the liquid penetrates inside the polymer matrix. When liquid penetrates inside the fibrous membrane, fibers are swelling and changing the volume which causes more solvent penetration but also it can be easily displaced by a squeezing tissue since it is weakly held in fibrous membrane. In the case of alcohol, it swells the polymer but also evaporates quickly, therefore, the found values are lower than the real values. In the case oil adsorption, especially silicon oil, less volatility, high viscosity and affinity of oils to each other help to remain more on the membrane surface as well as inside the fibrous membrane. Furthermore, the adsorption kinetic was aimed to study using different solvents and oils but adsorption occurs immediately in the first a couple of seconds, therefore, we did not manage to study adsorption kinetic using kinetic equations. However, the fast adsorption was displayed by supporting videos using DMSO and silicon oil drops on fibrous membranes in supporting videos 1 (DMSO) and 2 (silicon oil).

\section{Conclusion}

The PIM-2 fibrous membrane was successfully produced by electrospinning technique. Structural characterization and the purity of the membranes were confirmed by FT-IR, NMR and XPS spectroscopies. Bead-free and uniform fibers were obtained with an average diameter of $5.5 \pm 1.5 \mu \mathrm{m}$. Thermal properties, porosity and wettability of the PIM-2 fibrous membrane was measured to investigate structure-property relationship. High thermal stability and high surface area are provided by the rigid and contorted structure of PIM-2. In addition, high fluorine content and fibrous structure make polymer superhydrophobic with a water contact angle of $155 \pm 6^{\circ}$. With these combined properties, self-standing fibrous membrane of PIM-2 has shown considerable organic solvents and oil uptake up to 22 times increase in membrane weight. Also, dense membrane form of PIM-2 was prepared and employed in the same applications. Compared to fibrous membrane, smaller pores resulted in lower adsorption capacity in dense membranes. The successful preparation of such PIM membranes may provide new insights into the design and development of functional membranes for wastewater treatment for several other applications including VOC capture and catalysis.

\section{Appendix A. Supplementary material}

Supplementary data to this article can be found online at https:// doi.org/10.1016/j.eurpolymj.2018.12.029.

\section{References}

[1] P.M. Budd, B.S. Ghanem, S. Makhseed, N.B. McKeown, K.J. Msayib, C.E. Tattershall, Polymers of intrinsic microporosity (PIMs): robust, solution-processable, organic nanoporous materials, Chem. Commun. 2 (2004) 230-231.

[2] P.M. Budd, K.J. Msayib, C.E. Tattershall, B.S. Ghanem, K.J. Reynolds, N.B. McKeown, D. Fritsch, Gas separation membranes from polymers of intrinsic microporosity, J. Membr. Sci. 251 (1-2) (2005) 263-269.

[3] P.M. Budd, N.B. McKeown, D. Fritsch, Free volume and intrinsic microporosity in polymers, J. Mater. Chem. 15 (20) (2005) 1977-1986.

[4] F.Y. Li, Y. Xiao, T.S. Chung, High-performance thermally self-cross-linked polymer of intrinsic microporosity (PIM-1) membranes for energy development, Procedia Eng. 44 (2012) 498-500.

[5] S. Tsarkov, V. Khotimskiy, P.M. Budd, V. Volkov, J. Kukushkina, A. Volkov, Solvent nanofiltration through high permeability glassy polymers: effect of polymer and solute nature, J. Membr. Sci. 423-424 (2012) 65-72.

[6] N. Du, J. Song, G.P. Robertson, I. Pinnau, M.D. Guiver, Linear high molecular weight ladder polymer via fast polycondensation of 5, $5^{\prime}, 6,6^{\prime}$-tetrahydroxy-3,3, $3^{\prime}, 3^{\prime}$ tetramethylspirobisindane with 1,4-dicyanotetrafluorobenzene, Macromol. Rapid Commun. 29 (10) (2008) 783-788.

[7] B. Satilmis, P.M. Budd, Selective dye adsorption by chemically-modified and thermally-treated polymers of intrinsic microporosity, J. Colloid Interface Sci. 492 (2017) 81-91.

[8] P.M. Budd, N.B. McKeown, Highly permeable polymers for gas separation membranes, Polym. Chem. 1 (1) (2010) 63-68.

[9] D. Fritsch, P. Merten, K. Heinrich, M. Lazar, M. Priske, High performance organic solvent nanofiltration membranes: development and thorough testing of thin film composite membranes made of polymers of intrinsic microporosity (PIMs), J. Membr. Sci. 401-402 (2012) 222-231.

[10] M. Žák, M. Klepic, L.Č. Štastná, Z. Sedláková, H. Vychodilová, Š. Hovorka, K. Friess, A. Randová, L. Brožová, J.C. Jansen, M.R. Khdhayyer, P.M. Budd, P. Izák, Selective removal of butanol from aqueous solution by pervaporation with a PIM-1 membrane and membrane aging, Sep. Purif. Technol. 151 (2015) 108-114.

[11] B. Satilmis, T. Uyar, Removal of aniline from air and water by polymers of intrinsic microporosity (PIM-1) electrospun ultrafine fibers, J. Colloid Interface Sci. 516 (2018) 317-324.

[12] M.L. Jue, V. Breedveld, R.P. Lively, Defect-free PIM-1 hollow fiber membranes, J. Membr. Sci. 530 (Supplement C) (2017) 33-41.

[13] L.M. Robeson, The upper bound revisited, J. Membr. Sci. 320 (1) (2008) 390-400.

[14] D. Ramimoghadam, E.M. Gray, C.J. Webb, Review of polymers of intrinsic microporosity for hydrogen storage applications, Int. J. Hydrogen Energy 41 (38) (2016) 16944-16965.

[15] N.B. McKeown, P.M. Budd, Polymers of intrinsic microporosity (PIMs): organic materials for membrane separations, heterogeneous catalysis and hydrogen storage, Chem. Soc. Rev. 35 (8) (2006) 675-683.

[16] M. Carta, K.J. Msayib, N.B. McKeown, Novel polymers of intrinsic microporosity (PIMs) derived from 1,1-spiro-bis(1,2,3,4-tetrahydronaphthalene)-based monomers, Tetrahedron Lett. 50 (43) (2009) 5954-5957.

[17] S. Makhseed, F. Ibrahim, J. Samuel, Phthalimide based polymers of intrinsic microporosity, Polymer 53 (14) (2012) 2964-2972.

[18] J. Vile, M. Carta, C.G. Bezzu, B.M. Kariuki, N.B. McKeown, Centrotriindane- and triptindane-based polymers of intrinsic microporosity, Polymer 55 (1) (2014) 326-329.

[19] H. Sato, S. Nakajo, Y. Oishi, Y. Shibasaki, Synthesis of linear polymer of intrinsic microporosity from 5,5',6,6'-tetrahydroxy-3,3,3',3'-tetramethylspirobisindane and decafluorobiphenyl, React. Funct. Polym. 125 (2018) 70-76.

[20] S. Ramakrishna, K. Fujihara, W.-E. Teo, T.-C. Lim, Z. Ma, An Introduction to Electrospinning and Nanofibers, World Scientific, 2005.

[21] N. Bhardwaj, S.C. Kundu, Electrospinning: a fascinating fiber fabrication technique, Biotechnol. Adv. 28 (3) (2010) 325-347.

[22] T. Uyar, E. Kny, Electrospun Materials for Tissue Engineering and Biomedical Applications: Research, Design and Commercialization, Elsevier, Woodhead Publishing, 2017. 
[23] V. Shabafrooz, M. Mozafari, D. Vashaee, L. Tayebi, Electrospun nanofibers: From filtration membranes to highly specialized tissue engineering scaffolds, J. Nanosci. Nanotechnol. 14 (1) (2014) 522-534.

[24] X. Wang, B.S. Hsiao, Electrospun nanofiber membranes, Curr. Opin. Chem. Eng. 12 (2016) 62-81.

[25] Y. Ertas, T. Uyar, Fabrication of cellulose acetate/polybenzoxazine cross-linked electrospun nanofibrous membrane for water treatment, Carbohydr. Polym. 177 (2017) 378-387.

[26] O. Arslan, Z. Aytac, T. Uyar, Superhydrophobic, hybrid, electrospun cellulose acetate nanofibrous mats for oil/water separation by tailored surface modification, ACS Appl. Mater. Interfaces 8 (30) (2016) 19747-19754.

[27] B. Satilmis, T. Uyar, Superhydrophobic hexamethylene diisocyanate modified hy drolyzed polymers of intrinsic microporosity electrospun ultrafine fibrous membrane for the adsorption of organic compounds and oil/water separation, ACS Appl. Nano Mater. 1 (4) (2018) 1631-1640.

[28] K.S. Ranjith, B. Satilmis, T. Uyar, Hierarchical electrospun PIM nanofibers decorated with $\mathrm{ZnO}$ nanorods for effective pollutant adsorption and photocatalytic degradation, Mater. Today 21 (9) (2018) 989-990.

[29] K. Halder, G. Bengtson, V. Filiz, V. Abetz, Catalytically active (Pd) nanoparticles supported by electrospun PIM-1: influence of the sorption capacity of the polymer tested in the reduction of some aromatic nitro compounds, Appl Catal A-Gen 555 (2018) 178-188.

[30] J.S. Bonso, G.D. Kalaw, J.P. Ferraris, High surface area carbon nanofibers derive from electrospun PIM-1 for energy storage applications, J. Mater. Chem. A 2 (2) (2014) 418-424.

[31] B.S. Lalia, E. Guillen-Burrieza, H.A. Arafat, R. Hashaikeh, Fabrication and characterization of polyvinylidenefluoride-co-hexafluoropropylene (PVDF-HFP) electrospun membranes for direct contact membrane distillation, J. Membr. Sci. 428 (2013) 104-115.

[32] Y. Zhang, B. Yang, K. Li, D. Hou, C. Zhao, J. Wang, Electrospun porous poly(tetrafluoroethylene-co-hexafluoropropylene-co-vinylidene fluoride) membranes for membrane distillation, RSC Adv. 7 (89) (2017) 56183-56193.

[33] X. Li, M.C. García-Payo, M. Khayet, M. Wang, X. Wang, Superhydrophobic polysulfone/polydimethylsiloxane electrospun nanofibrous membranes for water desalination by direct contact membrane distillation, J. Membr. Sci. 542 (2017) 308-319.

[34] P. Li, T.S. Chung, D.R. Paul, Gas sorption and permeation in PIM-1, J. Membr. Sci. 432 (2013) 50-57.

[35] P. Bernardo, E. Bazzarelli, F. Tasselli, G. Clarizia, C.R. Mason, L. Maynard-Atem, P.M. Budd, M. Lanc, K. Pilnacek, O. Vopicka, K. Friess, D. Fritsch, Y.P. Yampolskii, V. Shantarovich, J.C. Jansen, Effect of physical aging on the gas transport and sorption in PIM-1 membranes, Polymer 113 (2017) 283-294.

[36] R.R. Tiwari, J.Y. Jin, B.D. Freeman, D.R. Paul, Physical aging, CO2 sorption and plasticization in thin films of polymer with intrinsic microporosity (PIM-1), J.
Membr. Sci. 537 (2017) 362-371.

[37] M. Minelli, K. Friess, O. Vopicka, M.G. De Angelis, Modeling gas and vapor sorption in a polymer of intrinsic microporosity (PIM-1), Fluid Phase Equilib. 347 (2013) 35-44.

[38] B. Satilmis, P.M. Budd, T. Uyar, Systematic hydrolysis of PIM-1 and electrospinning of hydrolyzed PIM-1 ultrafine fibers for an efficient removal of dye from water, React. Funct. Polym. 121 (Supplement C) (2017) 67-75.

[39] B. Satilmis, T. Uyar, Amine modified electrospun PIM-1 ultrafine fibers for an efficient removal of methyl orange from an aqueous system, Appl. Surf. Sci. 453 (2018) 220-229.

[40] C. Zhang, P. Li, B. Cao, Electrospun microfibrous membranes based on PIM-1/POSS with high oil wettability for separation of oil-water mixtures and cleanup of oil soluble contaminants, Ind. Eng. Chem. Res. 54 (35) (2015) 8772-8781.

[41] E. Lasseuguette, M.-C. Ferrari, Development of microporous electrospun PIM-1 fibres, Mater. Lett. 177 (2016) 116-119.

[42] Y. Pan, L.J. Zhang, Z.J. Li, L.J. Ma, Y.F. Zhang, J. Wang, J.Q. Meng, Hierarchical porous membrane via electrospinning PIM-1 for micropollutants removal, Appl. Surf. Sci. 443 (2018) 441-451.

[43] B. Satilmis, T. Isık, M.M. Demir, T. Uyar, Amidoxime functionalized Polymers of Intrinsic Microporosity (PIM-1) electrospun ultrafine fibers for rapid removal of uranyl ions from water, Appl. Surf. Sci. 467-468 (2019) 648-657.

[44] B. Satilmis, P.M. Budd, Base-catalysed hydrolysis of PIM-1: amide versus carboxylate formation, RSC Adv. 4 (94) (2014) 52189-52198.

[45] S.V. Adymkanov, Y.P. Yampol'skii, A.M. Polyakov, P.M. Budd, K.J. Reynolds, N.B. McKeown, K.J. Msayib, Pervaporation of alcohols through highly permeable PIM-1 polymer films, Polym. Sci. Series A 50 (4) (2008) 444-450.

[46] Y. Wang, N.B. McKeown, K.J. Msayib, G.A. Turnbull, I.D.W. Samuel, Laser chemosensor with rapid responsivity and inherent memory based on a polymer of intrinsic microporosity, Sensors (Basel, Switzerland) 11 (3) (2011) 2478-2487.

[47] F.Y. Li, T.-S. Chung, Physical aging, high temperature and water vapor permeation studies of UV-rearranged PIM-1 membranes for advanced hydrogen purification and production, Int. J. Hydrogen Energy 38 (23) (2013) 9786-9793.

[48] T.S. Anokhina, A.A. Yushkin, P.M. Budd, A.V. Volkov, Application of PIM-1 for solvent swing adsorption and solvent recovery by nanofiltration, Sep. Purif. Technol. 156 (2015) 683-690.

[49] H.J. Kim, D.G. Kim, K. Lee, Y. Baek, Y. Yoo, Y.S. Kim, B.G. Kim, J.C. Lee, A Carbonaceous Membrane based on a Polymer of Intrinsic Microporosity (PIM-1) for Water Treatment, Sci. Rep. 6 (2016).

[50] X.S. Zhao, Q. Ma, G.Q. Lu, V.O.C. Removal, Comparison of MCM-41 with hydrophobic zeolites and activated carbon, Energy Fuels 12 (6) (1998) 1051-1054.

[51] F.D. Yu, L.A. Luo, G. Grevillot, Adsorption isotherms of VOCs onto an activated carbon monolith: experimental measurement and correlation with different models, J. Chem. Eng. Data 47 (3) (2002) 467-473. 